\title{
Supporting information part
}

Fully biosourced materials from combination of choline-chloride-based deep eutectic solvents and guar gum.

Jérémy Depoorter, ${ }^{\mathrm{a}}$ Adeline Mourlevat, ${ }^{\mathrm{a}}$ Guillaume Sudre, ${ }^{\mathrm{b}}$ Isabelle Morfin, ${ }^{\mathrm{c}}$ Kamalesh Prasad, ${ }^{\text {de }}$ Anatoli Serghei, ${ }^{\mathrm{b}}$ Julien Bernard, ${ }^{\mathrm{a}}$ Etienne Fleury ${ }^{\mathrm{a} *}$, Aurélia Charlot ${ }^{\mathrm{a}}$

a Laboratoire Ingénierie des Matériaux Polymères, UMR CNRS 5223, INSA Lyon, Bâtiment Jules Verne, 17 avenue Jean Capelle, 69621 Villeurbanne, France.

b Laboratoire Ingénierie des Matériaux Polymères, UMR CNRS 5223, Université Lyon 1, 15 Bd. A. Latarjet, 69621 Villeurbanne, France.

${ }^{\mathrm{c}}$ Université Grenoble Alpes, CNRS, LiPhy, 38000 Grenoble, France

d Natural Product \& Green Chemistry Division, CSIR, Central Salt and Marine Chemicals Research Institue (CSIR-CSMCRI), G.B. Marg, Bhavnagar, 364 002, India.

${ }^{\mathrm{e}}$ Academy of Scientific and Innovative Research (AcSIR), Ghaziabad- 201002, India.

*Corresponding authors: etienne.fleury@insa-lyon.fr, aurelia.charlot@insa-lyon.fr

Number of pages: $11 \quad$ Number of figures: 13 Number of Table: 1 


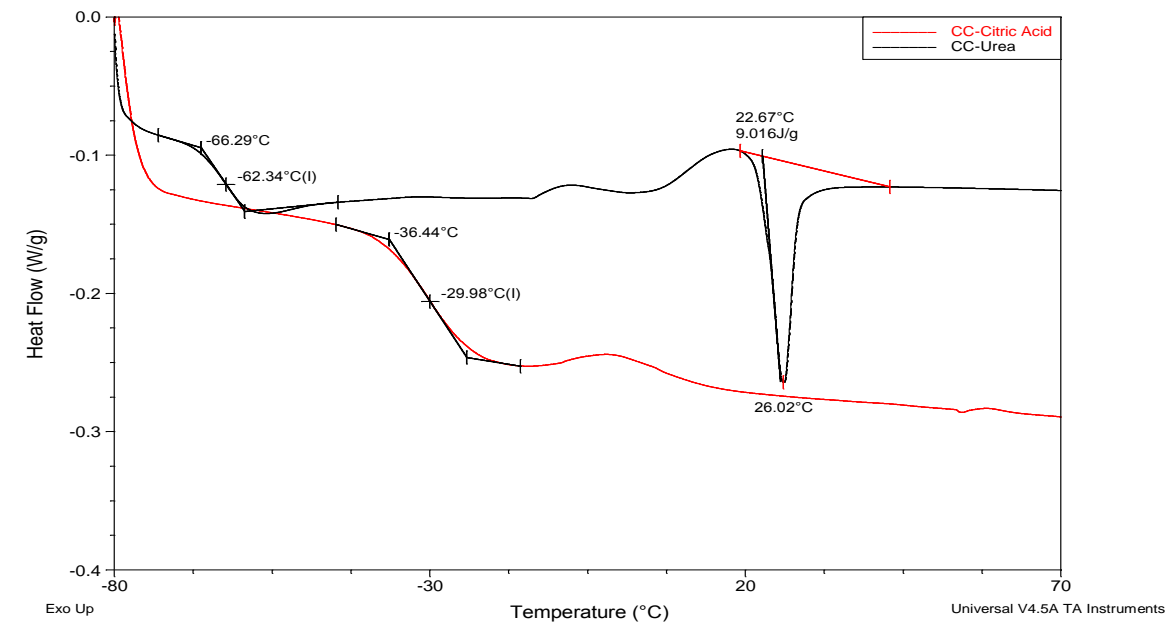

Figure S1. Differential Scanning Calorimetry (DSC) of ChCl-Urea and ChCl-Citric acid.

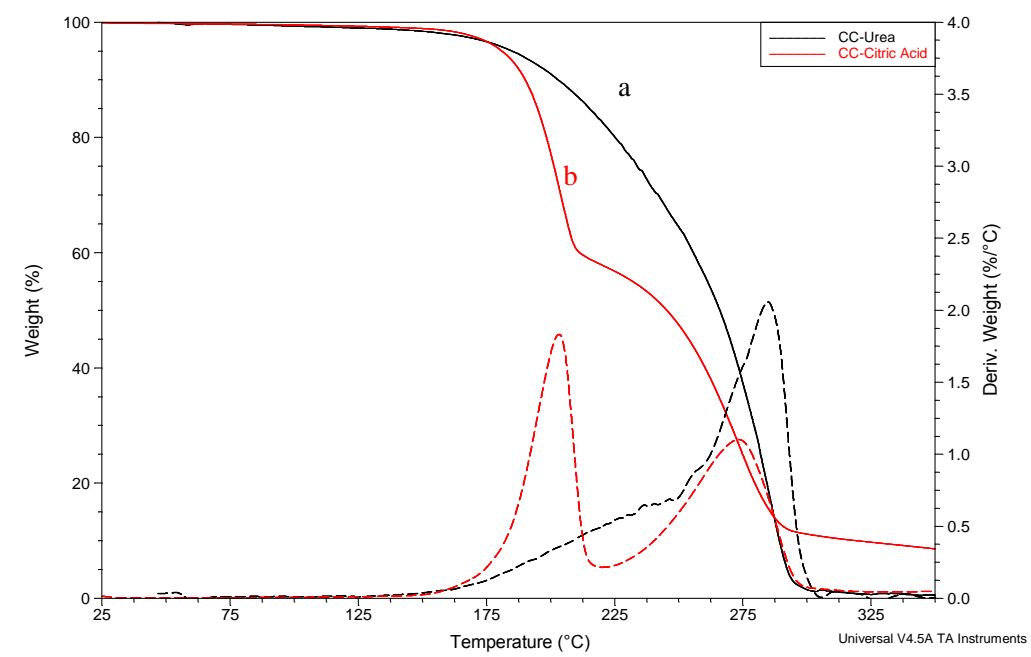

Figure S2. TGA of ChCl-Urea (a) and ChCl-Citric acid (b). 


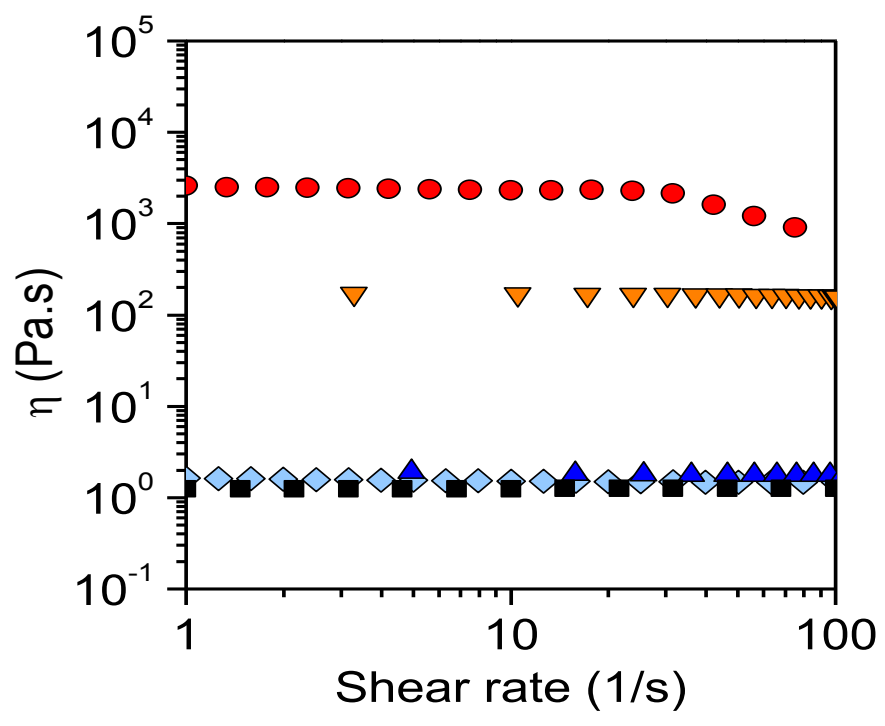

Figure S3. Shear rate-dependence of viscosity of $\mathrm{ChCl} / \mathrm{Urea}(\mathbf{\bullet}), \mathrm{ChCl} / \mathrm{Citric}$ acid (•),

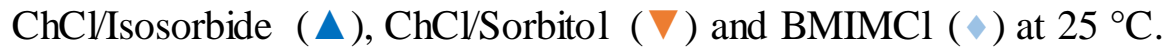



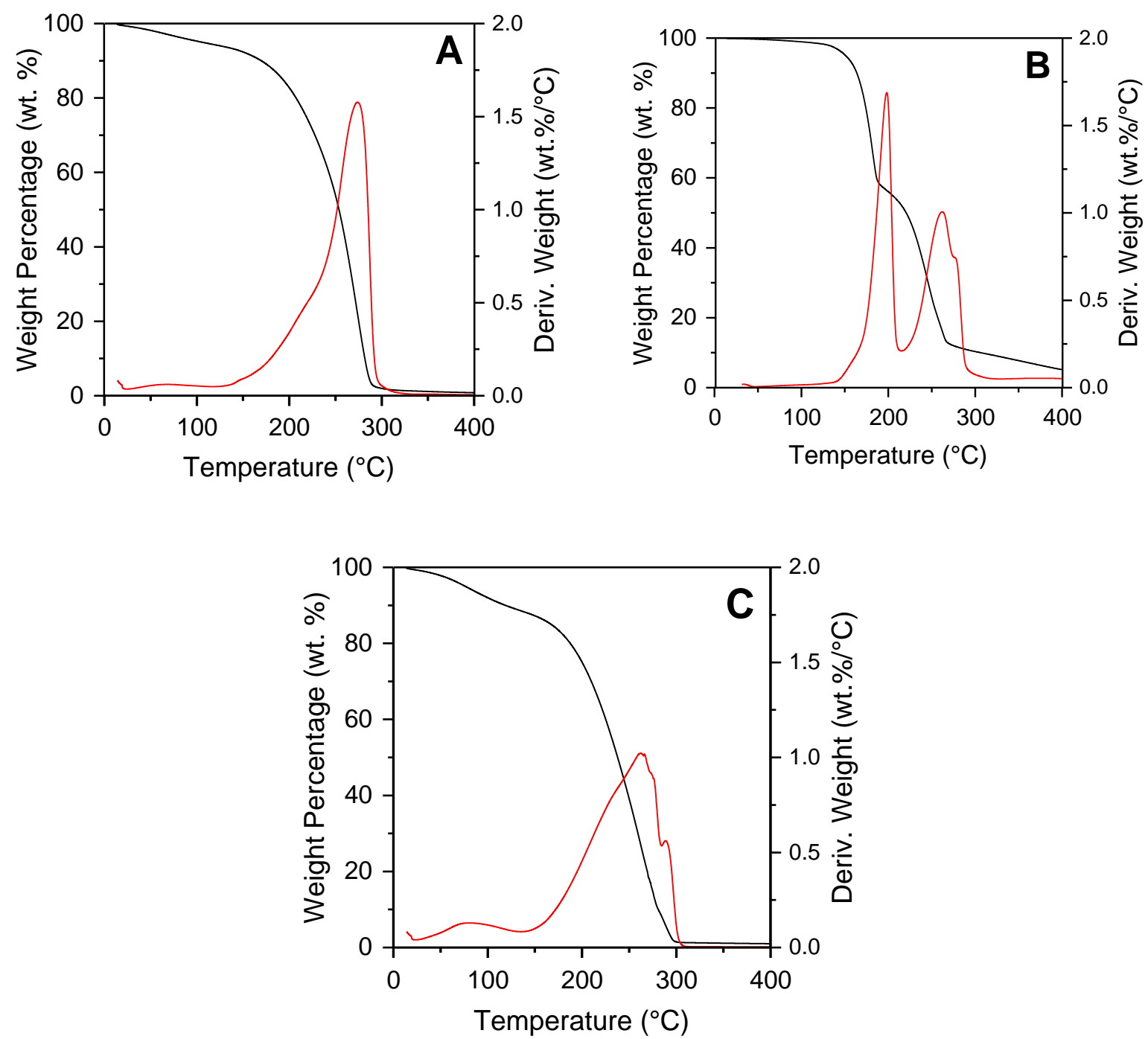

Figure S4. TGA of ChCl-Urea (Figure A), ChCl/Citric acid (Figure B), and ChCl/Isosorbide (Figure C), after storage during 6 months. 

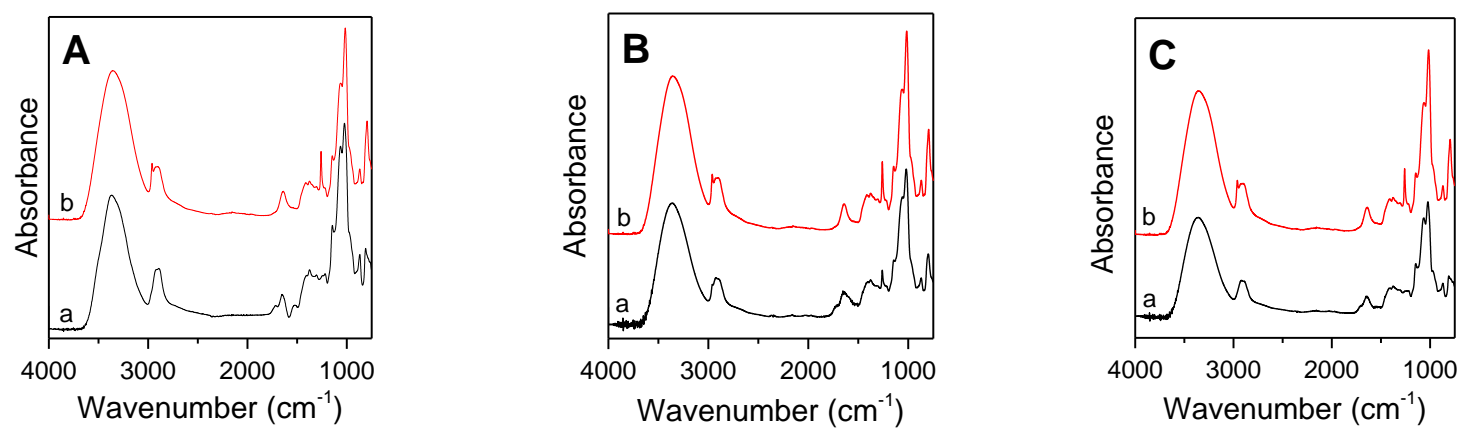

Figure S5. FT-IR spectra of guar regenerated from mixture with ChCl-Urea (Figure A b)), from mixture with $\mathrm{ChCl}$-Citric acid (Figure $\mathrm{B} \mathrm{b}$ )), and from mixture with $\mathrm{ChCl}$-Isosorbide (Figure $\mathrm{C}$ c)), compared to native guar subjected to a hydration/freeze-drying step (spectra a).

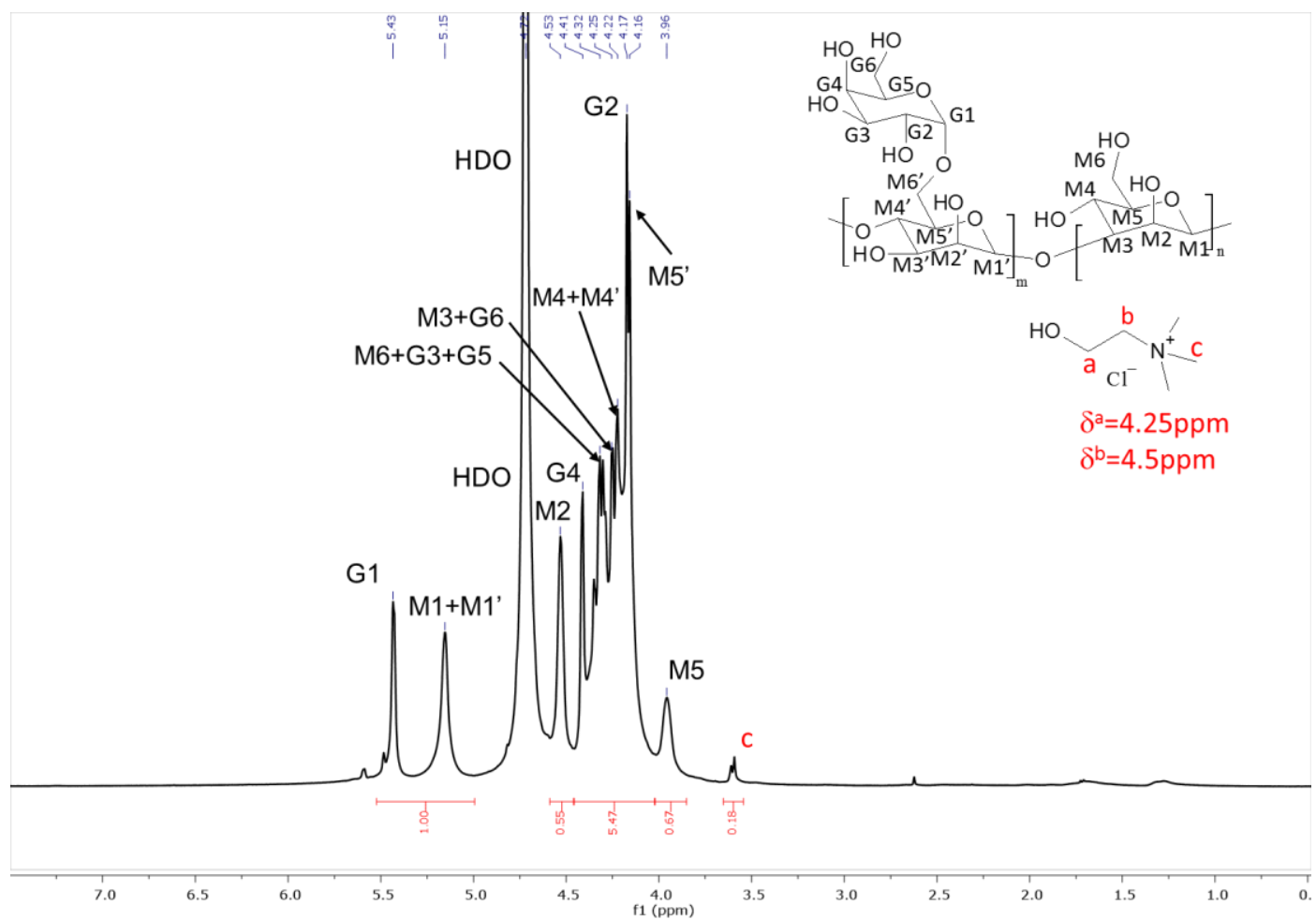

Figure S6. ${ }^{1} \mathrm{H}$ NMR spectrum of guar regenerated from mixture with $\mathrm{ChCl}-\mathrm{Urea}\left(\mathrm{C}_{\text {guar }}=10\right.$ wt. $\%$ in $\mathrm{D}_{2} \mathrm{O}$ at $343 \mathrm{~K}, 512$ scans). 


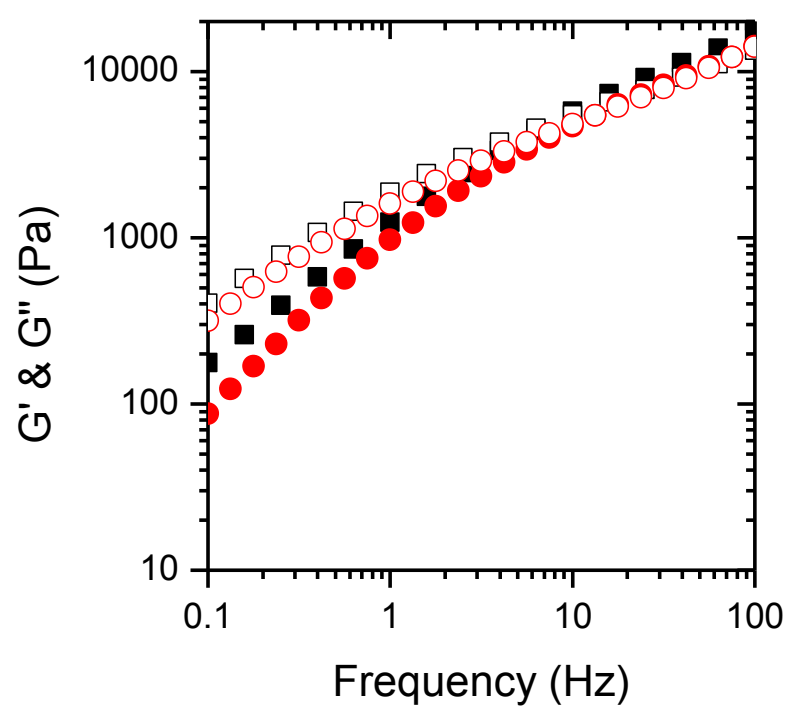

Figure S7. Frequency-dependence of G' (filled symbols) and G', (open symbols) moduli at $25^{\circ} \mathrm{C}$ of $\mathrm{Guar} / \mathrm{ChCl}-$ Urea $\mathrm{C}_{\text {guar }}=10 \mathrm{wt}$. $\%$, by using native guar $(\mathbf{a})$ and regenerated guar from a mixture with $\mathrm{ChCl}$-Urea $(\bullet)$. 

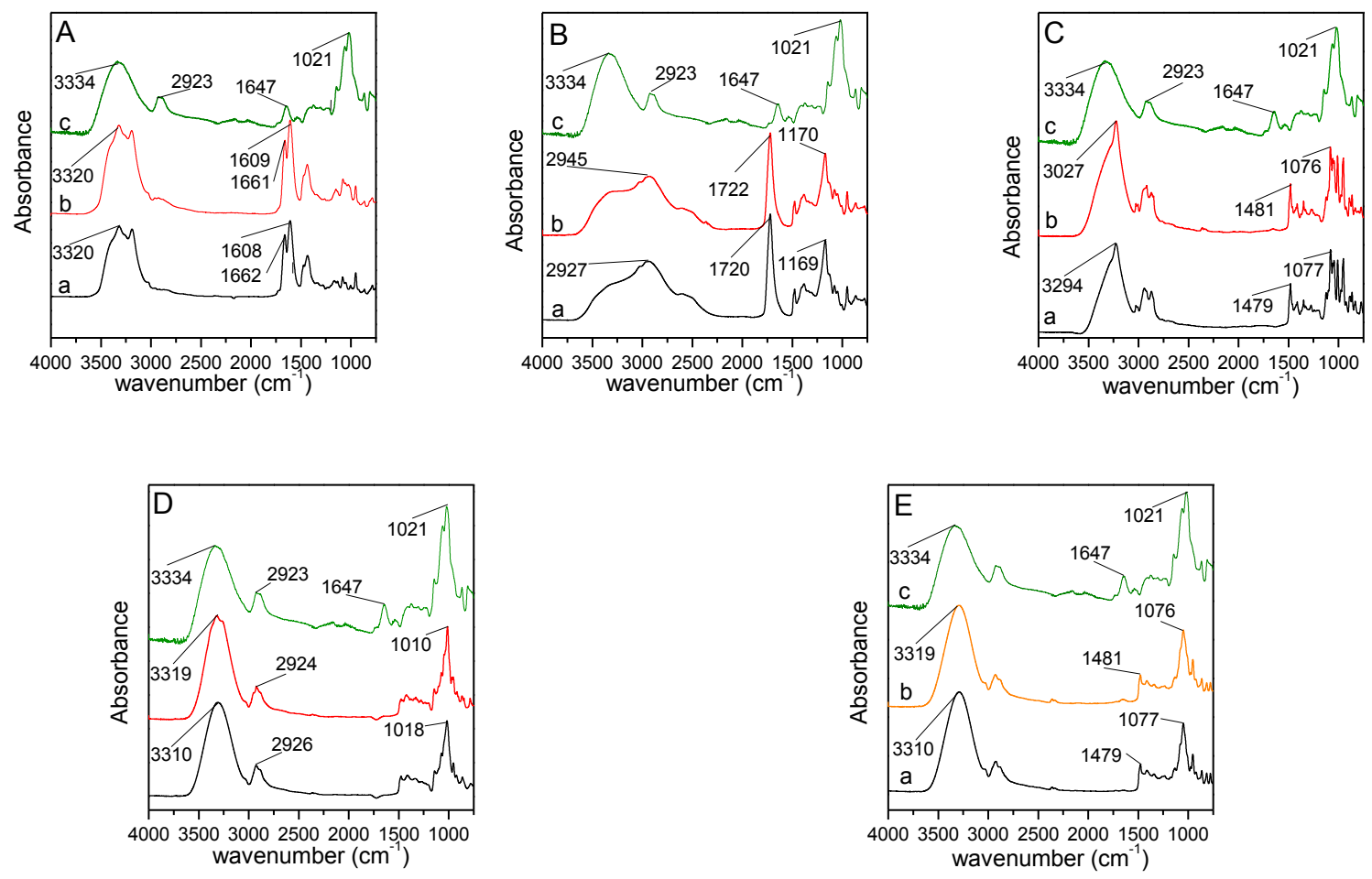

Figure S8. FT-IR spectra of ChCl-Urea (a), Guar/ChCl-Urea (C=10 wt. \%) (b) and guar (c) (Figure A)); ChCl-Citric acid (a), Guar/ChCl-Citric acid (C = 10 wt. \%) (b) and guar (c) (Figure B)); ChCl-Isosorbide (a), Guar/ChCl-Isosorbide (C = $10 \mathrm{wt} . \%)$ (b) and guar (c) (Figure C)); ChCl-Sorbitol (a), Guar/Sorbitol (C = $10 \mathrm{wt} . \%$ ) (b) and guar (c) (Figure D)); ChCl-Fructose (a), Guar/ChCl-Fructose (C=5 wt. \%) (b) and guar (c) (Figure E)). . 

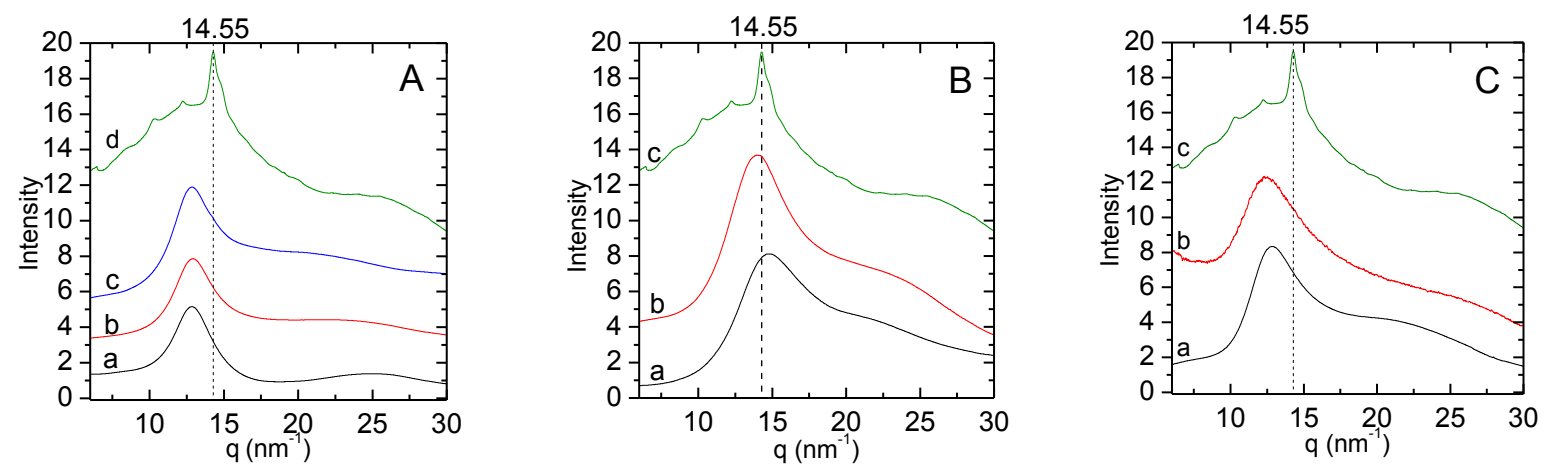

Figure S9. WAXS patterns at $25{ }^{\circ} \mathrm{C}$ of Guar/ChCl-Isosorbide (a), Guar/ChCl-Isosorbide at $\mathrm{C}_{\text {guar }}=10 \mathrm{w} \%$ (b), $20 \mathrm{w} \%$ (c), and native guar (d) (Figure A); ChCl-Sorbitol (a), Guar/ChClSorbitol at $\mathrm{C}_{\text {guar }}=10 \mathrm{wt} \%$ (b), and native guar (c) (Figure A); ChCl-Fructose (a), Guar/ChClFructose at $\mathrm{C}_{\text {guar }}=10 \mathrm{wt}$ \% (b), and native guar (c) (Figure $\mathrm{C}$ ).
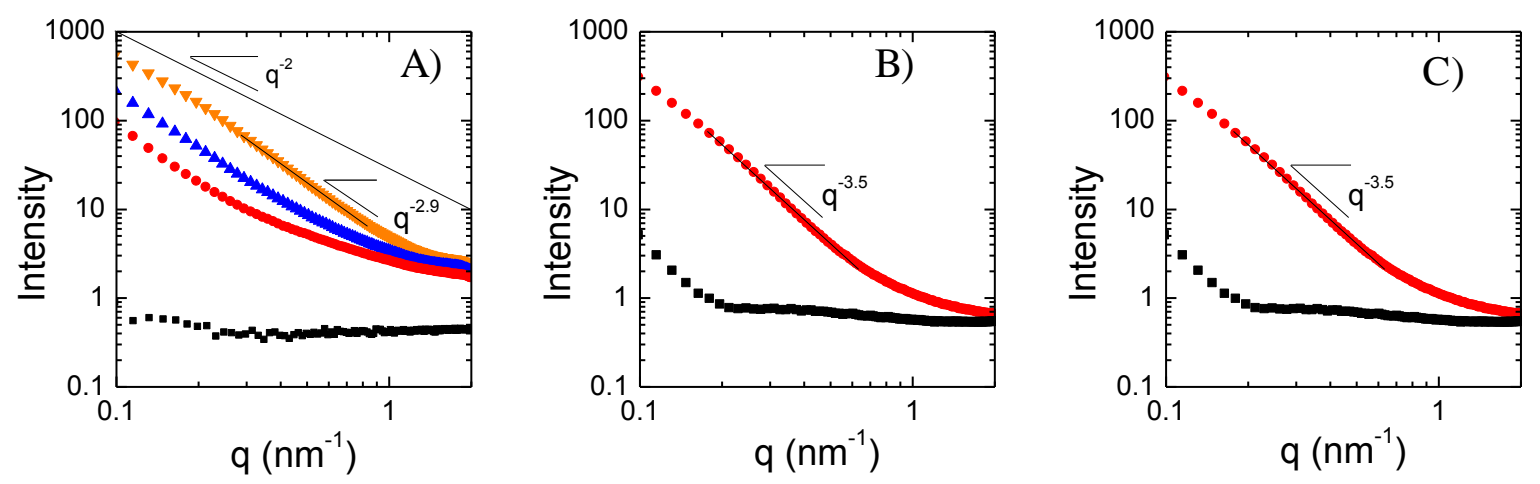

Figure S10. SAXS patterns of ChCl-Urea (घ), Guar/ChCl-Urea at $\mathrm{C}_{\text {guar }}=20 \mathrm{w} \%(\bullet), 30$

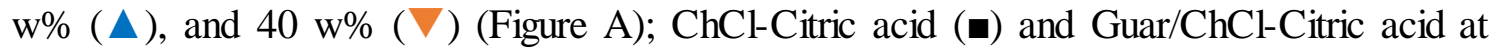
$\mathrm{C}_{\text {guar }}=20 \mathrm{w} \%(\bullet)$ (Figure B); C) ChCl-Isosorbide (匚), and Guar/ChCl-Isosorbide at $\mathrm{C}_{\text {guar }}=20 \mathrm{w} \%(\bullet)$ (Figure C). 


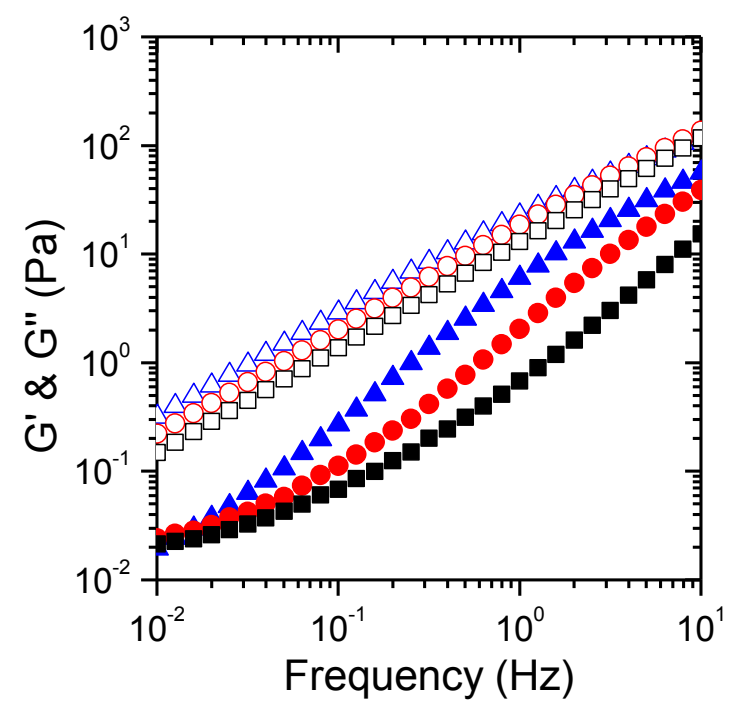

Figure S11. Frequency-dependence of G' (filled symbols) and G', (open symbols) moduli at $25^{\circ} \mathrm{C}$ of Guar/ChCl-Urea $\left(\mathrm{C}_{\text {guar }}=1\right.$ wt. \%) with a guar molecular weight of $119000 \mathrm{~g} / \mathrm{mol}$ (匹), $200000 \mathrm{~g} / \mathrm{mol}(\bullet)$ and $421000 \mathrm{~g} / \mathrm{mol}(\Delta)$.

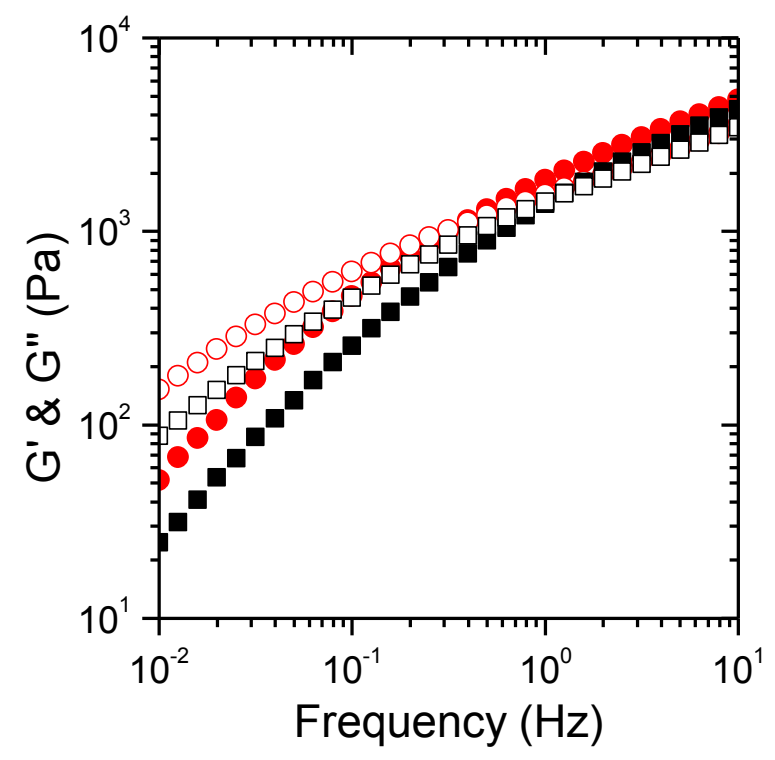

Figure S12. Frequency-dependence of G' (filled symbols) and G', (open symbols) moduli at $25^{\circ} \mathrm{C}$ of Guar/ChCl-Urea ( $\bullet$, and Guar/BMIMCl ( $\bullet$ ) for $\mathrm{C}_{\text {guar }}=7.5$ wt. $\%$. 

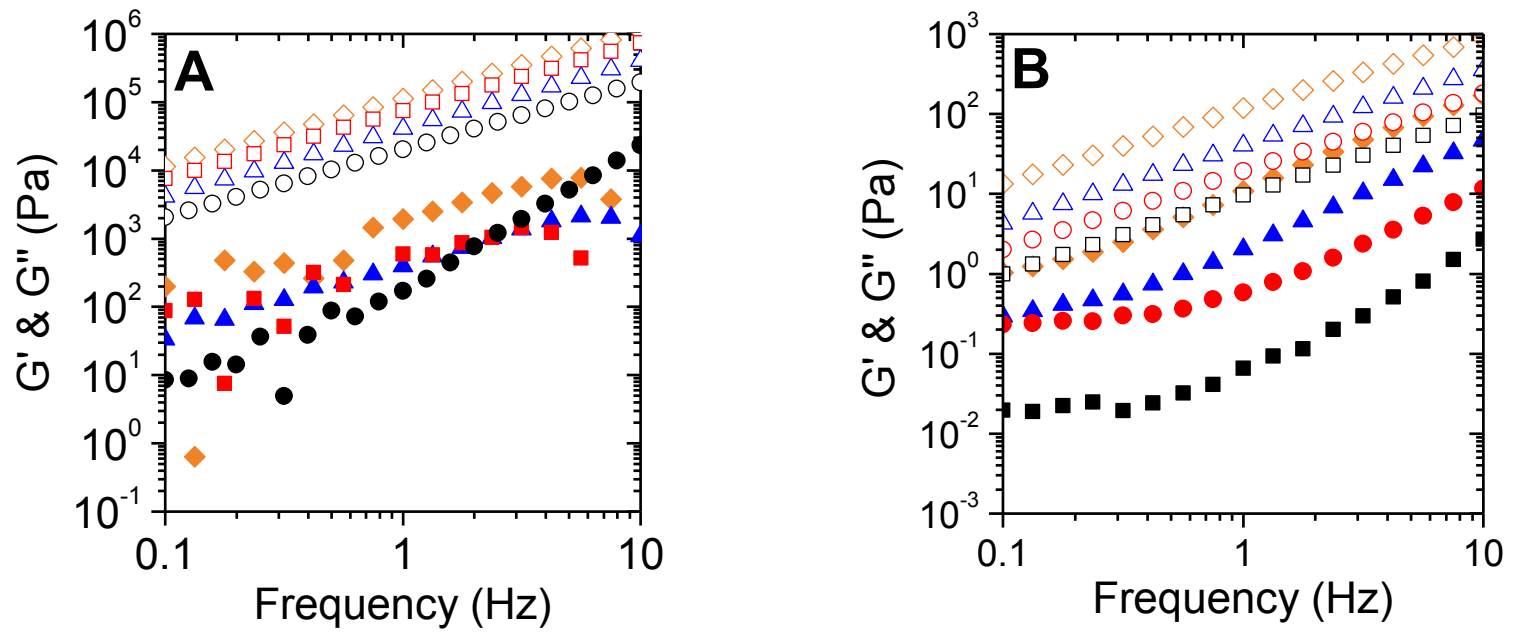

Figure S13.Frequency-dependence of G' (filled symbols) and G' (open symbols) moduli of ChCl-Citric acid ( $\mathbf{a})$ and guar/ChCl-Citric acid at $\mathrm{C}_{\text {guar }}=5 \mathrm{w} \%(\bullet), 10 \mathrm{w} \%(\boldsymbol{\Delta})$ and $20 \mathrm{w} \%$ (४) (Figure A), ChCl-Isosorbide (๘) and guar/ChCl-Isosorbide at $\mathrm{C}_{\text {guar }}=5 \mathrm{w} \%(\bullet), 10 \mathrm{w} \%$ $(\Delta)$ and $20 \mathrm{w} \%(\diamond)$ (Figure B). 
Table S1. VFT parameters of various samples.

\begin{tabular}{|c|c|c|c|}
\hline Samples & $\sigma_{0}(\mathrm{~S} / \mathrm{cm})$ & $\mathbf{B}(\mathbf{K})$ & $\mathbf{T}_{\mathbf{0}}(\mathbf{K})$ \\
\hline BMIMCl & 18.7 & 1575 & 170 \\
\hline ChCl-Urea & 9.9 & 1413 & 173 \\
\hline $\begin{array}{l}\mathrm{Guar} / \mathrm{BMIMCl}\left(\mathrm{C}_{\text {guar }}=10 \mathrm{wt} . \% \text { ) }\right. \\
\text { (before thermal treatment) }\end{array}$ & 8.6 & 1398 & 170 \\
\hline $\begin{array}{l}\text { Guar/ChCl-Urea }\left(\mathrm{C}_{\text {guar }}=10\right. \\
\text { wt. } \%) \quad(\text { after } \quad \text { thermal } \\
\text { treatment) }\end{array}$ & 12 & 1354 & 180 \\
\hline Guar/ChCl-Urea $\left(\mathrm{C}_{\text {guar }}=20\right.$ wt. \% $)$ & 10.0 & 1622 & 180 \\
\hline
\end{tabular}

